\title{
Measurand-Optimized, Content-Aware Scanning Electron Microscopy
}

\author{
András E. Vladár ${ }^{1}$ and Bradley N. Damazo ${ }^{1}$ \\ ${ }^{1 .}$ Microsystems and Nanotechnology Division National Institute of Standards and Technology (NIST)*, \\ MD, USA.
}

The performance of the best scanning electron microscopes (SEMs) today allows for measurements with sub-nanometer resolution. To take advantage of their excellent sub- $0.5 \mathrm{~nm}$ focusing ability, one must acquire images the fastest way possible to minimize problems arising from sample drift, charging, contamination, damage, vibration, etc. A $1 \mathrm{~nm}$ diameter Si sphere has only 26 atoms in a volume of about $0.4 \mathrm{~nm}^{3}$. Some of the best, model-based size and shape SEM measurements of $10 \mathrm{~nm}$ size objects have achieved $0.1 \mathrm{~nm}$ or so measurement uncertainty associated with only a few atoms.[1] Clearly, a few atoms cannot produce a strong signal, so measuring nano-meter sized objects or features of larger structures requires a fully optimized SEM with much better acquisition methods than the ones available today. Many aspects of SEM measurements shall be improved to enable atomic resolution and to reach the ultimate, physics limits of scanning electron microscopy. One of the possibilities to significantly improve the SEM performance for nm-scale measurements lies in its scanning method.

Traditional raster scanning of the focused primary beam in the SEM can be wasteful, inescapably forcing the user to acquire information that is not needed or already known. In many SEMs the beam scans a larger area over the sample, than the acquired image. This so-called over-scan has been used in early and many modern SEMs to minimize distortions caused by coil-based beam deflection systems. Another reason was that the beam to generate images with less influence of AC line voltage, the vertical and horizontal deflection signals are synchronized with the phase of 50 or $60 \mathrm{~Hz}$ line voltage. The over-scan can reach $10 \%$ or more of the time of the useful image frame. See Figure 1. Over-scan is not needed in SEMs with electrostatic beam deflection, especially if they are kept contamination-free. [2,3]

Another reason for the raster scanning method being inefficient is that it disregards the image content, the fact that some of the pixels of the SEM image contain information represented by more electrons (brighter) than others. For example, the gold particles of Figure 2 are a lot brighter and their signal-to-ratio (SNR) is better than of the polystyrene particles. The generated signals in the SEM have shot (Poisson) noise with a SNR of $\sqrt{n}$ (for $n$ detected electrons), so to achieve sufficient SNR, more time is needed for lowintensity pixels, than for high ones. It is better to spend the acquisition time on improving the SNR at lowintensity pixels than unnecessarily measuring again and again those that have appropriate SNR already. Non-raster, smart steering of the primary beam could significantly speed up acquisition time while optimizing SNR.

Another possibility for significant speed improvement is a different, measurand-dependent, fully optimized beam scanning. This new scanning method, instead of spending time acquiring unnecessary information in a whole image frame, scans only the pixels that are required to measure a given measurand with the desired uncertainty. For example, if particle perimeter is the measurand, after quickly determining the locations of the particles, the beam scans the fastest way only across the edges of the particles, not wasting time with scanning over areas that do not contain any information about the measurand, see Figure 2 insert at the lower left corner. Depending on the measurand, desired uncertainty, signal yield, charging, etc. further optimization is possible. The fully optimized beam scanning could make measurements faster, 
even by more than an order of magnitude, it could make (for example, for sample damage reason) otherwise not possible measurements feasible [4].

\section{References:}

[1] Vladar et al., $10 \mathrm{~nm}$ 3-dimensional CD-SEM metrology (2014) https://doi.org/10.1117/12.2045977

[2] Scipioni et al., Helium Ion Microscope Advanced Materials and Processes (2008).

[3] Vladar et al., Elimination of Electron-Beam-Induced Carbonaceous Contamination in SEMs and the new RGM 10100 NIST Contamination Testing Artifact (2018)

https://www2.avs.org/symposium2018/Papers/Paper_VT-TuP9.html

[4] Contributions of the National Institute of Standards and Technology are not subject to copyright. Mention of commercial products or services in this paper does not imply approval or endorsement by NIST, nor does it imply that such products or services are necessarily the best available for the purpose.

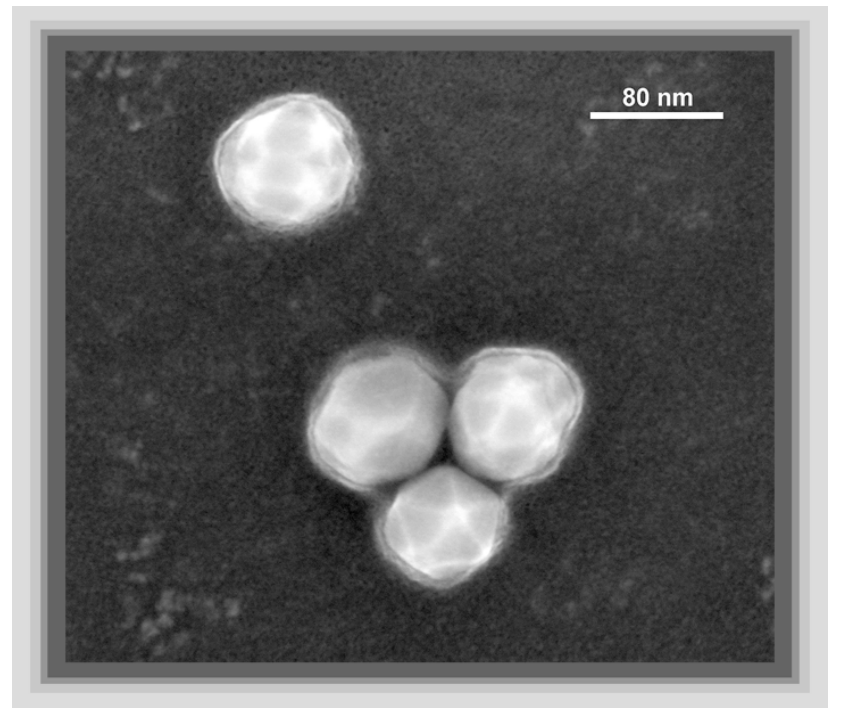

Figure 1. Full area scan of a typical 1024 by 884 pixel image, with the over-scan of $5 \%$ (1075 by 928), $7 \%$ (1096 by 928$), 10 \%$ (1126 by 972 and $15 \%$ (1178 by 1017$)$ means $10 \%, 14 \%, 21 \%$ and $32 \%$ unused time for imaging. $429 \mathrm{~nm}$ horizontal field width, $15 \mathrm{keV}, 86 \mathrm{pA}$

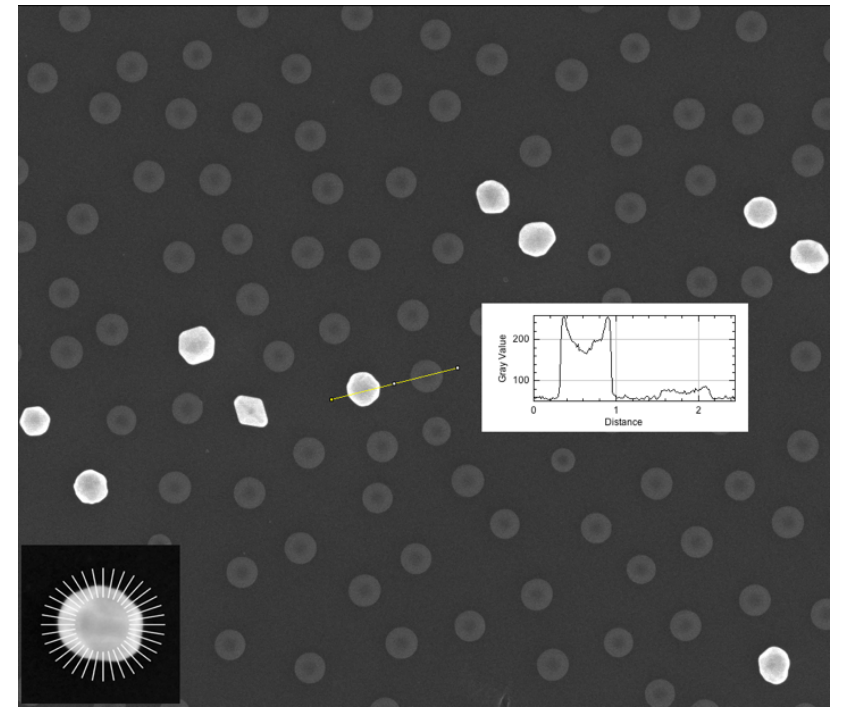

Figure 2. Traditional raster scanning image of bright gold and faint polystyrene particles with different peak signal intensity and hence SNR. Lower left insert is an illustration of measurand-optimized non-raster scanning of a particle. $2540 \mathrm{~nm}$ horizontal field width, $5 \mathrm{keV}$, $21 \mathrm{pA}$ 